\title{
Depression and African Americans: Developing a Culturally Competent Minority Depression and Ways of Coping Scale
}

\author{
Kennedy BR* \\ Senior Consultant, Global Healthcare Consulting Firm, LLC, USA
}

*Corresponding author: Dr. Bernice Roberts Kennedy, Senior Consultant, Researcher, BRK Global Healthcare Consulting Firm, LLC, USA, Email: brkhealthcare@gmail.com

\section{Investigation Paper}

Volume 5 Issue 1

Received Date: December 16, 2020

Published Date: January 04, 2021

DOI: $10.23880 /$ phoa-16000168

\section{Abstract}

Often, African Americans are misdiagnosed and under-treated in the conventional healthcare system. Research studies have reported the symptoms of depression among African Americans are inconsistent with the DSM-V. Previous Depression scales have not been culturally competent reflecting the depression symptoms of African Americans. The researcher developed a depression scale to reflect the racism and psychosocial factors contributing to depression in African Americans. In the future, the researcher will use the revised depression scale on African Americans to further investigate the symptoms of depression in African Americans. If depression is adequately identified in African Americans, health providers can accurately diagnose depression and provide culturally competent treatment.

Keywords: African Americans; Depression; Depression Scale; Ways of Coping; Cultural Competence

\section{Introduction}

Researchers need to develop more reliable, practical, and valid tools to measure mental health in African Americans and other minority groups [1]. Tools for assessing depression need to be culturally sensitive. Also, healthcare professionals need to be aware of cultural factors when conducting assessments and obtaining a medical history and physical (H\&P). When prescribing psychotropic medications clients need to be adequately assessed and provided the correct diagnosis [2]. Also, clients need to be accurately diagnosed when providing culturally competent therapy [1]. African Americans experience numerous socioeconomic differences in comparison to the Majority race [3]. The Diagnostic and Statistical Manual of Mental Disorders (DSM-V) acknowledges that clinicians should take culture and socioeconomic factors under consideration when diagnosing [4]. When diagnosing African Americans, the clinicians may have certain cultural bias and the cultural differences may be misinterpreted [1,3]. A clinician must be sensitive to the norms and values in the African American culture. The current DSM-V tried including a section on cultural and diagnosis [4]. However, African Americans continue to be over-diagnosed with as schizophrenia. The difference of African Americans in expressing their symptoms may contribute to their misdiagnosed [5]. Often, African Americans are misdiagnosed and under-treated in the conventional healthcare system [6]. A holistic approach is useful for African Americans in reducing depression this approach encompasses the biopsychosocial-spiritual aspects of their treatment. African Americans are more likely to be undiagnosed and to experience more severe or persistent depression [1]. Also, African American women are more likely to report somatic complaints and sleep and appetite changes $[1,7,8]$. Whereas White Americans are more likely to have more cognitive disturbances such as indecisiveness and worthlessness [7]. African Americans aged 15 to 24 years are more likely to report physical health problems than emotional instability [9]. 
In an ethnographic study by Alang [10] of African Americans in a disadvantaged urban neighborhood located in the Midwestern of the United States, participants identify meanings and commons ways of expressing depression. Depression was often considered a sign of weakness rather than a health problem that might need medical attention. However, the researcher reported that the signs of depression such as sadness and hopelessness were inconsistent with notions of strength. In the DSM-V common indicators of depression included symptoms such as hopelessness, loss of sleep, and feeling worthless. However, the expressions of depression such as anger, agitation, violent behavior, and a frantic search for social interaction are inconsistent with DSM-V criteria. These findings indicated a need for culturally competent in the diagnosis of African Americans and the need for better diagnosing and assessment tools.

\section{B.R. Kennedy Culturally Sensitive Depression Scale for Minority Groups (Original Scale)}

The BR Kennedy Culturally Sensitive Depression Scale for Minority Groups was first developed in 2009 [11]. The scale was developed by Dr. Bernice Roberts Kennedy based on 150 African Americans' responses to reports of depression in focus groups. Then, a comprehensive review of scholarly literature and research studies was conducted on African Americans and depression to assist with developing this scale. This scale focused on the cultural factors, psychosocial factors, and coping factors contributing to depression in African Americans. The original scale was a 25 -item selfreported inventory and is ideally suited based on its unique culture-sensitive items.

The original scale BR Kennedy Culturally Sensitive Depression Scale for Minority Groupswasused in a dissertation on Rural Obese African American Women and Depression, Food Culture, and Binge Eating [12]. The participants in this study consist of $12(17.4 \%)$ African American males and 57 (82.6\%) African American females. For all subjects, this scale was used to measure self-reported perceptions of depression. Also, this 25-item Likert scale was used to report depression based on these categories: 0-25 (normal); 26-50 (mild mood changes); 51-75 (moderate depression); and 76-100 (severe depression). In this study, the Kennedy Depression Inventory had a Cronbach alpha of 0.90 . The respondents reported an estimated degree of depression severity as follows: 39 (60\%) respondents showed normal depressive states; 22 (33.9\%) showed mild depression, 3 (4.6\%) had moderate depression, and $(1.5 \%)$ showed a severe depression. In responding to Question 23 of BR Kennedy Culturally Sensitive Depression Scale Depression Scale, $88.5 \%$ of the participants responded that would pray or attend religious services; and in Question
$25,98.5 \%$ answered that they would continue to pray or be encouraged despite being depressed.

\section{B.R. Kennedy Culturally Sensitive Depression and Ways of Coping Inventory Scales (Revised Scale)}

B. R. Kennedy Culturally Sensitive Depression Scale for Minority Groups. was revised in 2017 based on the question items such as the use of prayer, church attendance, religious involvement, regular church attendance, and even dressing up when feeling depressed by African American women [12]. These are common coping mechanisms for African Americans

The revised scale was renamed as the B.R. Kennedy Culturally Sensitive Depression and Ways of Coping Inventory Scales. The scale consists of 25 items in symptoms of depression in the Depression Inventory section and 13 items in the Ways of Coping section. The scale worded the questions to reflect the racism and psychosocial factors contributing to depression in African Americans. In the Depression Inventory section of this scale, there are 17 items on psychosocial symptoms and 8 items focusing on the physical symptoms. The Ways of Coping section of scale includes 13 items in response to coping with depression to include effective coping and ineffective coping. For example, effective coping (resiliency) includes prayer, religious involvement, family support, etc. and ineffective coping includes anger, violent behavior, the use of substances, etc.

\section{Conclusion}

In the future, the researcher will use the revised scale on African Americans to further investigate the symptoms of depression in African Americans and other ethnic minority groups. If depression is adequately identified in African Americans, health providers can accurately diagnose and provide culturally competent treatment. The researcher would like to investigate depression in African Americans living in different areas of the United States, other counties, different age groups, and a larger sample size.

\section{Acknowledgment}

I like to thank Dr. Tracee Tamiko Smith for using the original scale in her dissertation at the School of Public Health, Walden University; Minneapolis, MN.

\section{References}

1. Kennedy BR, Rhodes CC (2018) African American women and depression. Promoting the need for culturally 


\section{Public Health Open Access}

competent treatment. BRK Global Healthcare Journal 2(1): 1-26.

2. Lawson W, Johnston S, Karson C, Offord S, Docherty J, et al. (2015) Racial differences in antipsychotic use: Claims database analysis of Medicaid-insured patients with schizophrenia. Ann Clin Psychiatry 27(4): 242-252.

3. Horton SM (2014) Exploring the Pathological Label of Blacks: An African-Centered Exploration. Exploring the Pathological Label of Blacks, pp: 1-20.

4. APA (2013) Diagnostic and Statistical Manual of Mental Disorders, $5^{\text {th }}$ (Edn.), American Psychiatric Association, Washington, DC, USA.

5. Ramsey JM, Cooper JD, Bot M, Guest PC, Lamers F, et al. (2016) Sex differences in serum makers of major depressive disorders in the Netherlands study of depression and anxiety (NESDA). PLos One 11(5): e0156624.

6. Sohail Z, Bailey RK, Richlie WD (2014) Misconceptions of depression in African Americans. Front Psychiatry 5: 65.

7. Hankerson SH, Fenton MC, Geier TJ, Keves KM,
Wesissman MM, et al. (2011) Racial difference in symptoms, comorbidity, and treatment for major depressive disorder among black and white adults. J Natl Med Assoc 103(7): 576-584.

8. Sandraluz LC, Akinbode TD, Wood J (2020) A systematic review of somatic symptoms in women with depression or depressive symptoms: Do race or ethnicity matter. J Women's Health 29(10): 1273-1282.

9. Bailey R, Blackmon H, Stevens F (2009) Major depressive disorder in the African American population: Meeting the challenges of stigma, misdiagnoses, and treatment disparities. J Natl Med Assoc 101(11): 1084-1089.

10. Alang S (2016) Black Folk Don't Get any Severe Depression: Meanings and Expressions of Depression in a Predominantly Black Urban Neighborhood in Midwestern United States. Soc Sci Med 157: 1-8.

11. Kennedy BK (2009) Depression and African American women. Iuniversi Publication.

12. Smith TT (2016) Rural Obese African American Women and Depression Food, Culture, and Binge Eating, Walden University, pp: 1-134. 